\title{
The First Harmonic of Radial Pulse as an Early Predictor of Silent Coronary Artery Disease and Adverse Cardiac Events in Type 2 Diabetic Patients
}

\author{
Chi-Wei Chang $\mathbb{D}^{1}{ }^{1}$ Kuo-meng Liao, ${ }^{2}$ Yi-Ting Chang, ${ }^{3}$ Sheng-Hung Wang, \\ Ying-chun Chen, ${ }^{2}$ and Gin-Chung Wang ${ }^{5}$ \\ ${ }^{1}$ Graduate Institute of Biomedical Electronics and Bioinformatics, National Taiwan University, Taipei, Taiwan \\ ${ }^{2}$ Division of Endocrinology and Metabolism of Zhongxiao Branch of Taipei City Hospital, Taipei, Taiwan \\ ${ }^{3}$ Biostatistics, Johns Hopkins Bloomberg School of Public Health, Baltimore, USA \\ ${ }^{4}$ Metal Industries Research \& Development Centre, Kaohsiung, Taiwan \\ ${ }^{5}$ JinMu Health Technology, Taipei, Taiwan \\ Correspondence should be addressed to Chi-Wei Chang; s750711@gmail.com
}

Received 5 July 2018; Revised 11 September 2018; Accepted 23 September 2018; Published 23 October 2018

Guest Editor: Kirsten Riches-Suman

Copyright (c) 2018 Chi-Wei Chang et al. This is an open access article distributed under the Creative Commons Attribution License, which permits unrestricted use, distribution, and reproduction in any medium, provided the original work is properly cited.

\begin{abstract}
Background. It has been reported that harmonics of radial pulse is related to coronary artery disease (CAD) in patients with type 2 diabetes mellitus (T2DM). It is still unclear whether or not the first harmonics of the radial pulse spectrum is an early independent predictor of silent coronary artery disease (SCAD) and adverse cardiac events (ACE). Objectives. To measure the risk of SCAD in patients with T2DM and also to survey whether or not an increment of the first harmonic $(C 1)$ of the radial pulse increases ACE. Methods. 1968 asymptomatic individuals with T2DM underwent radial pulse wave measurement. First harmonic of the radial pressure wave, $C 1$, was calculated. Next, the new occurrence of ACE and the new symptoms and signs of coronary artery disease were recorded. The follow-up period lasted for $14.7 \pm 3.5$ months. Results. Out of 1968 asymptomatic individuals with T2DM, ACE was detected in 239 (12\%) of them during the follow-up period. The logrank test demonstrated that the cumulative incidence of ACE in patients with $C 1$ above 0.96 was greater than that in those patients with $C 1$ below $0.89(P<0.01)$. By comparing the data of patients with $C 1$ smaller than the first quartile and the patients with $C 1$ greater than the third quartile, the hazard ratios were listed as follows: ACE (hazard ratio, 2.29; 95\% CI, 1.55-3.37), heart failure (hazard ratio, 2.22; 95\% CI, 1.21-4.09), myocardial infarction (hazard ratio, 2.44; 95\% CI, 1.51-3.93), left ventricular dysfunction (Hazard ratio, 2.01; 95\% CI, 0.86-4.70), and new symptoms and signs for coronary artery disease (hazard ratio, 2.03; 95\% CI, 1.45-2.84). As C1 increased, the risk for composite ACE $(P<0.001$ for trend) and for coronary disease $(P<0.001$ for trend $)$ also increased. The hazard ratio and trend for cardiovascular-cause mortality were not significant. Conclusions. This study showed that $C 1$ of the radial pulse wave is correlated with cardiovascular events. Survival analysis showed that $C 1$ value is an independent predictor of ACE and SCAD in asymptomatic patients with T2DM. Thus, screening for the first harmonic of the radial pulse may improve the risk stratification of cardiac events and SCAD in asymptomatic patients although they had no history of coronary artery disease or anginarelated symptom.
\end{abstract}

\section{Introduction}

Coronary artery disease (CAD) is one of the leading causes of death and contributes to the adverse cardiovascular events. Compared with nondiabetics, patients with type 2 diabetes mellitus (T2DM) have 2- to 4 -fold increase risk of CAD [1] and are more often without history and symptoms of coronary artery disease until the onset of myocardial infarction or sudden cardiac death [2]. An asymptomatic patient with T2DM has been shown $20-35 \%$ prevalence of 
silent CAD (SCAD) [3, 4], which could lead to myocardial ischemia, adverse cardiac events (ACE), and a poor prognosis outcome [5-9]. Hence, it is essential to have early predictors to screen the high-risk asymptomatic diabetic patients, to give a risk assessment of SCAD and ACE. Thus, diabetic patients with SCAD may benefit from screening and further medical intervention to prevent sudden cardiac death or adverse cardiovascular events.

Several studies made an effort on the identification of those diabetic patients with SCAD [10], using coronary artery calcium imaging or stress radionuclide myocardial perfusion imaging (MPI) [11-14]. However, the risk of the stress test and the radiation dose in those images makes the screening of diabetic patients with SCAD remain controversial. European society of cardiology guideline suggested class II to class III recommendation and was not used in patients with a low range of intermediate of pretest probability for CAD [15]. Therefore, the patients with diabetes and clinically at high risk of SCAD need to be further defined and investigated before calcium imaging score, MPI, or invasive coronary angiography (ICA) is performed.

The emerging technical method to screen SCAD is using the harmonic index in arterial pulse spectrum analysis. In the 1970s, medical researchers introduced harmonic analysis to convert pressure pulses and flow pulses into numerical Fourier series [16]. Harmonics is one of the complete quantitative descriptions of periodic pulse waveforms [17]. Initially, the harmonic analysis was applied to the transfer function between the aortic pulse wave and the radial pulse wave [18]. In recent decades, many studies have used harmonic analysis to assess vascular sclerosis [19], aortic occlusion [20], aging effects [21], vasodilator drugs [22], and heart diseases $[23,24]$. Chen et al. first found that the specific characteristic of radial pulse spectrum changed from the resting state to the onset of acute, uncomplicated myocardial infarction state, and gradually shifted to other resting characteristics a week after the surgery [25]. A study suggests that harmonic characteristics may be an adjunct method for identifying patients who require intensive treatment or revascularization $[26,27]$.

In addition, the important relationship between the first harmonic of the radial pulse wave and cardiac risk has been studied recently. Wang et al. demonstrated that the first harmonics of the radial pulse increased with age [21]. The cross-sectional cohort study showed that the first, second, and third harmonics of the radial pulse correlated with the ischemic heart symptoms [28]. The population mean of the first harmonic is also higher in asymptomatic diabetic patients with more than $5 \%$ of ischemic myocardium, compared with those without significant myocardial perfusion defects $(P<0.05)$ [29]. Since the first harmonic is associated with the aging process and with the signs and symptoms of myocardial ischemia, the next issue is whether the first harmonic of radial pulse is an independent predictor for SCAD and for adverse cardiac events (ACE). Therefore, this study aimed to statistically validate the degree of confidence that the first harmonic of radial pulse is associated with SCAD and ACE in a prospective cohort study, using survival analysis and Cox regression model.

\section{Methods}

2.1. Patients. The present population included 1158 men and 810 women who had a record of T2DM and have already entered a diabetes management program in the Division of Endocrinology \& Metabolism of Zhongxiao Branch of Taipei City Hospital. In order to investigate the asymptomatic patients, the key exclusion criteria were listed as follows: (1) angina pectoris or angina equivalent symptoms; (2) any stress test or coronary angiography before the enrollment; (3) shortness of breath either at rest or on exertion; (4) history of myocardial infarction, heart failure, or coronary revascularization; (5) electrocardiographic evidence of Q-wave abnormality, ischemic ST-segment, and T-wave abnormality; (6) clinically significant valvular heart disease or cardiomyopathy. Subjects were also excluded if the radial pulse wave measurement could not be carried out due to the severer diseases or acute symptoms such as end-stage renal disease or liver disease. A total of 1968 patients have been enrolled in the study between January 2017 and May 2018 in Taiwan. After the assessment of the first harmonic amplitude of the radial pulse wave at baseline, patients were followed for a mean of $14.7 \pm 3.5$ months. The study was approved by the Institutional Review Board of Taipei City Hospital (IRB number: ISRCTN14306167). Then, both oral and written information about the study will be given to the enrolled patients. All participants signed for their consent.

2.2. Study Design. The medical history and physical examination report were retrieved to determine the patient demographic data, the status of T2DM, and other clinical variables. Readings of radial pulse and harmonic analysis were taken in 1968 participants at baseline with a longest follow-up period that lasted for 16 months after the radial pulse measurement. Myocardial infarction, heart failure, and cardiovascular death were also documented.

The baseline of the first harmonics of the radial pulse was carried out at the beginning of the study. Each subject was required to lie down in a supine position and rest for 5 minutes before the measurement. The pulse pressure was then obtained on the left-hand radial artery with a pulse wave analyzer TD01C (MII-ANN Technology, Taiwan). TD01C has been proved its intrinsic reliability using an artificial pulse generator [30]. The intraobserver and interobserver reliabilities of TD01C were also manifested in the clinical study [31]. The successive pressure pulses were acquired during the 12 -second period. The pressure pulse data were recorded with a sampling rate of 400 data points per second. These data were then transformed into harmonic components $(\mathrm{Cn})$ and phase angle $(\mathrm{Pn})$ using Fourier transform. $C 1$ is defined by the following equation:

$$
C 1=\frac{A_{1}}{A_{0}}
$$

where $A_{0}$ is the mean value of pulse wave, and $A_{1}$ is the 1 st amplitude coefficient of Fourier series of the radial pulse wave. The mean value of $C 1$ calculated from all pulses was used as a representative harmonic amplitude value within 
one measurement. After the assessment of radial pulse spectrum, the blood pressure and heart rate were assessed by an automatic blood pressure monitor (HBP-9020, Omron, Japan) according to the instruction manual. The assessment was performed by a well-trained operator, without the doctors and nurse presented, to avoid the white coat effect.

2.3. Outcomes. The patients entering the diabetes management program will visit the research hospital every 4-6 months to conduct the clinical evaluation of cardiac risk, based on the risk factor assessment or cardiovascular events. The study used the risk factor evaluation to select the patients who need a further cardiac stress test such as SPECT myocardial perfusion image, or stress electrocardiogram (ECG), mostly following the guidelines $[24,25]$. The candidates for stress test are the patients with two or more following risk factors: (i) lipid disorders (total cholesterol $\geqq$ $240 \mathrm{mg} / \mathrm{dl}$, low-density lipoprotein (LDL) $\geqq 160 \mathrm{mg} / \mathrm{dl}$, or high-density lipoprotein (HDL) $\leqq 35 \mathrm{mg} / \mathrm{dl}$ ), (ii) blood pressure $>140 / 90 \mathrm{mmHg}$, (iii) family history of premature coronary heart disease, (iv) age $\geqq 70$ years, (v) newly discovered resting ECG abnormalities, (vi) ankle-brachial index $\leqq 0.9$, and (vii) albumin creatinine ratio $\geqq 30 \mathrm{mg} / \mathrm{g}$. After stress testing, revascularization will be considered in patients with moderate to severe myocardial ischemia (summed stress score $\geq 9$ ) or abnormal stress ECG.

The primary study outcome was a composite of myocardial infarction, heart failure, or cardiovascular mortality. The secondary outcome was a composite of left ventricular dysfunction (LVEF $\leq 50 \%$ ), and the new occurrence of symptoms and signs of coronary artery disease, which was defined by the related symptoms and signs of CAD diagnosed by the cardiology physicians.

Myocardial infarction was diagnosed by a cardiologist physician following the consensus guideline for Joint European Society of Cardiology/American College of Cardiology Committee [32] and the definition of World Health Organization, including a combination of at least two of three characteristics as follows:

(1) Enzyme rise: an increased value for cardiac troponin, exceeding the value of the 99th percentile of a reference control group, reflecting myocardial cell death.

(2) Abnormal electrocardiography: myocardial ischemia (ST-T segment changes) or evidence of loss of electrically functioning cardiac tissue (Q waves)

(3) The angina-related symptoms.

Heart failure was diagnosed by a cardiologist physician, including identification of a structural and functional abnormality, following the New York Heart Association (NYHA) Functional Classification and the guideline of American College of Cardiology [33, 34].

The signs of coronary artery disease included the newly diagnosed single coronary stenosis $(>70 \%)$, multicoronary stenoses $(>50 \%$ stenosis for at least two major coronary vessels), and more than mild myocardial ischemia using semiquantitative 20-segment analysis of myocardial perfusion single-photon emission computed tomography (GE Infinia, USA). Each of these outcomes was also analyzed separately. Source data were derived from the database of the diabetes management program in Zhongxiao Branch of Taipei City Hospital and were verified by independent monitors.

2.4. Statistical Analysis. The study was designed to follow the patients for 16 months. All the subjects were categorized by four quartiles of $C 1$ levels $(<0.89,0.89$ to $0.96,0.96$ to 1.07 , and $>1.07$ ), in which Cox proportional hazards model were adopted to compare hazard ratios of clinical events among quartile groups with reference to the first quartile $(C 1<$ 0.89 ). The unadjusted hazard ratio and $95 \%$ confidence intervals of all outcomes in each quartile were recorded. Tests for linear trend across quartiles were examined using Cox regression analysis. Linear trend tests were assessed both before and after adjusting for age, sex, smoking, systolic pressure, diastolic blood pressure, dyslipidemia, and Hbalc. The curves of Kaplan-Meier for primary and secondary composite endpoints are plotted in Figure 1 according to the quartile levels of $C 1$. In order to determine if $C 1$ has a significant effect on the risk of adverse heart events and loss of cardiac function, the logrank test was performed. Matlab version 9.2 (MathWorks Inc, USA) was used to conduct all statistical analyses.

\section{Results}

The baseline clinical characteristics of 1968 asymptomatic patients with T2DM were shown in Table 1. The mean age of participants was $62 \pm 12$ years, in which $58.8 \%$ of the participants were male. The mean systolic, diastolic blood pressure, and the Hbalc level were $128 \pm 12 \mathrm{mmHg}, 75 \pm$ $31 \mathrm{mmHg}$, and $7.0 \pm 1.1 \%$, respectively. Kaplan-Meier curve showed the primary and secondary composite endpoints of four groups, within 16 months follow-up. There was a graded relationship between the baseline $C 1$ and risk of ACE. The cumulative incidence of primary composite endpoint increased from $7.5 \%$ to $10.4 \%$ to $14 \%$ to $16.7 \%$ according to four quartiles of $C 1$ levels. This relationship extended into the heart function loss and CAD. The cumulative incidence of secondary composite endpoint increased from $10.8 \%$ to $16.5 \%$ to $16.3 \%$ to $24.1 \%$ according to the same four quartiles of $\mathrm{C} 1$ levels.

The listed incidence and the hazard ratios are written in Table 2. Compared with the first quartile $(C 1<0.89)$, the hazard ratio of primary composite endpoint for each quartile was 1.39 (95\% CI, $0.91-2.12$ ) for $C 1$ of 0.89 to $0.96,1.90$ (95\% CI, $1.28-2.84$ ) for $C 1$ of 0.96 to 1.07 , and 2.29 (95\% CI, $1.55-3.37)$ for $C 1>1.07$. The linear trends were significant for major adverse cardiac events before and after controlling for age, sex, smoking, systolic pressure, diastolic blood pressure, dyslipidemia, and Hbalc $(P<0.001)$.

Similar findings were also found in the hazard ratio of the secondary composite endpoint for each quartile: Compared with the first quartile $(C 1<0.89)$, the hazard ratio of secondary composite endpoint for each quartile were 1.56 


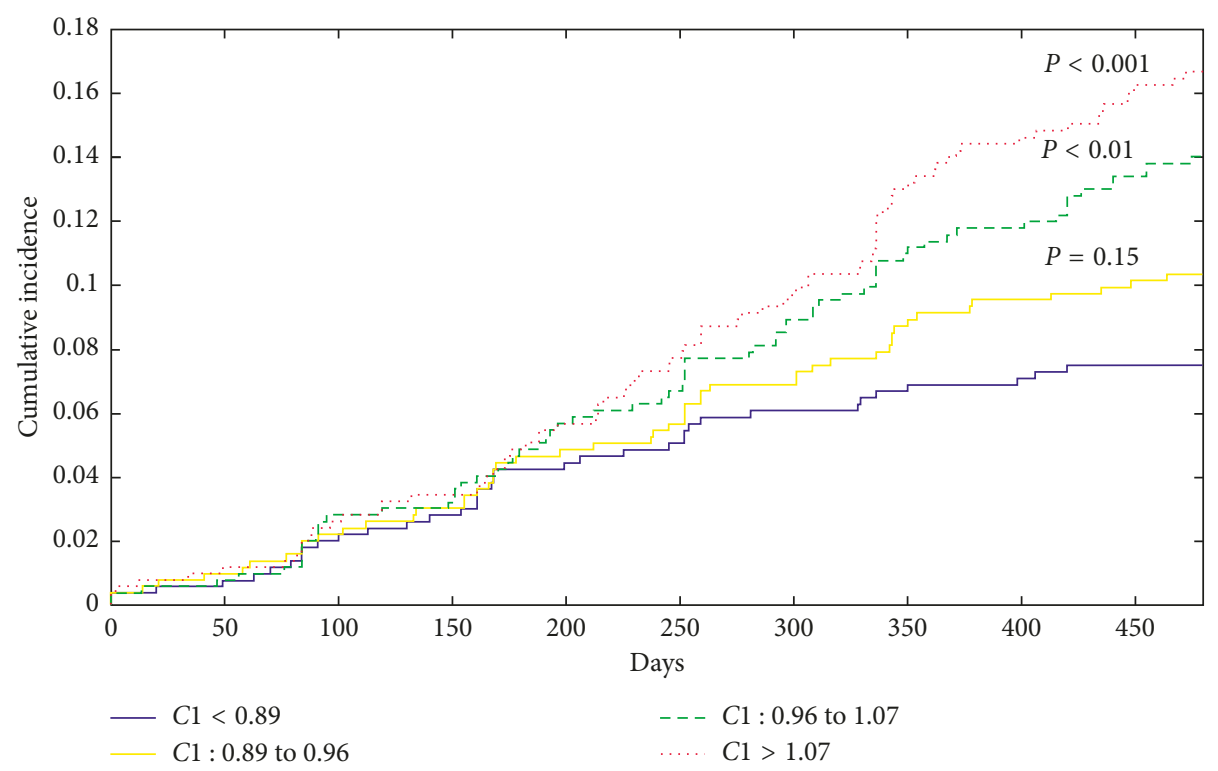

(a)

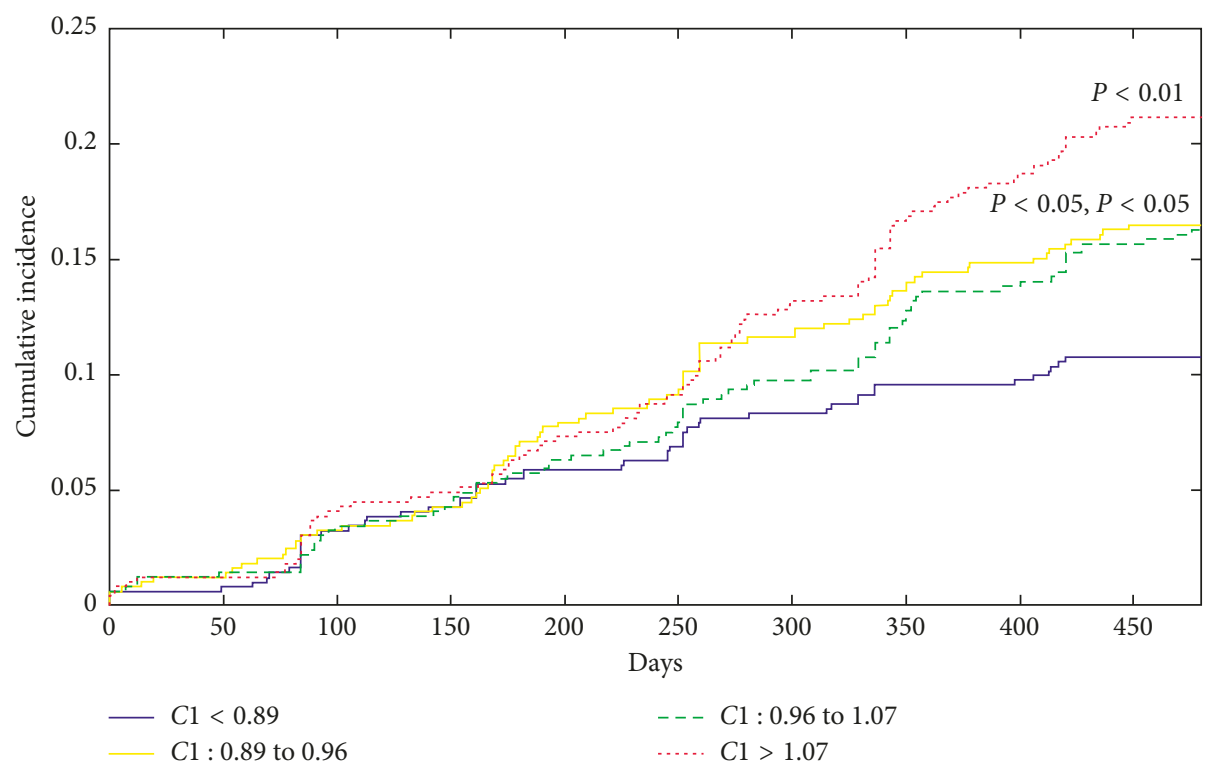

(b)

FIGURE 1: Kaplan-Meier event rates of the (a) primary and (b) secondary composite outcomes. Primary composite endpoints combined the adverse heart events, including new onset of heart failure, myocardial infarction, and cardiovascular mortality. Secondary composite endpoints combined the left ventricular dysfunction (LVEF $<50 \%)$ and the symptoms and signs of coronary artery disease $(n=1968) ; P$ values were the result of the logrank test. The reference group for logrank test is the first quartile of $C 1(<0.89)$.

(95\% CI, 1.11-2.21) for C1 of 0.89 to $0.96,1.53$ (95\% CI, $1.08-2.17$ ) for $C 1$ of 0.96 to 1.07 , and 2.04 (95\% CI, $1.46-$ 2.83 ) for $C 1>1.07$. The linear trends were significant for the symptoms and signs of CAD before and after controlling for age, sex, smoking, systolic pressure, diastolic blood pressure, dyslipidemia, and Hba1c $(P<0.001)$.

\section{Discussion}

The prevalence of SCAD in patients with T2DM varied from $10 \%$ to over $50 \%$ according to different study settings [35].
The diabetic patients with SCAD showed higher incidences of cardiovascular events and deaths compared with noSCAD diabetic patients using MPI [36-38], which indicated the need to identify the diabetic patients at high risk of SCAD. In asymptomatic patients with T2DM, whether carrying out the further investigation of CAD or not is a dilemma between the increasing risk of adverse heart disease and the increasing risk due to radiation exposure of coronary artery calcium imaging and due to the stress testing such as myocardial perfusion single-photon emission computed tomography. The European society of cardiology 
TABLE 1: Baseline clinical characteristics of the asymptomatic patients with type 2 diabetes.

\begin{tabular}{lc}
\hline Clinical characteristics & Value $(n=1968)$ \\
\hline Male $(\%)$ & 58.8 \\
Age (year) & $61.6 \pm 12.5$ \\
BMI $\left(\mathrm{kg} / \mathrm{m}^{2}\right)$ & $27.2 \pm 4.6$ \\
Waist circumference $(\mathrm{cm})$ & $93 \pm 12$ \\
Smoke $(\%)$ & 15.2 \\
SBP $(\mathrm{mmHg})$ & $128 \pm 12$ \\
DBP $(\mathrm{mmHg})$ & $74 \pm 8$ \\
PP (mmHg) & $53 \pm 10$ \\
HbA1C $(\%)$ & $7.0 \pm 1.1$ \\
EGFR (mL/min/1.73 $\left.\mathrm{m}^{2}\right)$ & $87 \pm 34$ \\
LDL (mg/dl) & $86 \pm 28$ \\
HDL (mg/dl) & $50 \pm 16$ \\
TC (mg/dl) & $160 \pm 34$ \\
TG (mg/dl) & $129 \pm 81$ \\
Heart rate (beats/min) & $73 \pm 11$ \\
Duration of diabetes (years) & $10 \pm 7$ \\
\hline
\end{tabular}

$\mathrm{SBP}=$ systolic blood pressure, $\mathrm{DBP}=$ diastolic blood pressure, $\mathrm{PP}=$ pulse pressure, $\mathrm{Hbalc}=$ glycated hemoglobin, $\mathrm{LDL}=$ low-density lipoprotein cholesterol, $\mathrm{HDL}=$ high-density lipoprotein cholesterol, $\mathrm{TC}=$ total cholesterol, $\mathrm{TG}=$ triglycerides, and EGFR $=$ estimated glomerular filtration rate.

guideline recommends not using those testing in patients with a low range of intermediate of pretest probability for CAD [15]. Therefore, without any history of CAD and angina-related symptoms, a proportion of the diabetic patients with SCAD were unrecognized until the onset of ACE $[3,4,39]$.

Since diabetes increases the cardiac risk, the need for new risk markers for SCAD exists. The patients with T2DM and at high risk of SCAD need to be further identified before calcium imaging score, MPI, or invasive coronary angiography (ICA) is performed. The ambulatory ECG has been demonstrated useful in predicting CAD and future cardiovascular event in asymptomatic diabetic patients [40]. However, ambulatory ECG had two limitations. First, ambulatory ECG was limited due to its lack of specificity, ranging from $50 \%-60 \%$ [41]. Second, there is no clear evidence for ambulatory ECG to provide reliable information about myocardial ischemia in asymptomatic patients, especially those without known CAD [42]. Also, Zellweger et al. combined several traditional risk factors of CAD to screen the asymptomatic high-risk patients with diabetes and to predict the abnormal score of MPI [43]. Anklebrachial index (ABI) has been proved to be associated with CAD [44-47], and low ABI was investigated as a predictor of CAD [48, 49]. Diabetic patients with microalbuminuria (albumin-to-creatinine ratio: $30-300 \mathrm{mg} / \mathrm{g}$ ) had also been confirmed with increased risk of CAD [50]. ABI measured a ratio of ankle systolic blood pressure to brachial systolic pressure and assessed the presence of occlusive peripheral arterial disease and generalized atherosclerosis [48, 51]. Both low ABI [52] and microalbuminuria [53-55] have been manifested in the detection of silent myocardial ischemia symptom.

Recently, the cross-sectional study screened the performance of $\mathrm{ABI}$, microalbuminuria, and harmonics of the radial pulse to stratify the risk of SCAD and severe silent myocardial ischemia in patients with T2DM and had at least one traditional risk factor. The harmonics of the radial pulse (ORs, 2.36-4.46), ABI (ORs, 2.24-6.90), and microalbuminuria (ORs, 0.5-1.70) showed different abilities to identify the patients at high risk of CAD [26]. Combining those risk markers using majority votes, the diagnostic performance for silent myocardial ischemia (area under ROC: 0.74) and for SCAD (Area under ROC: 0.82) was greatly improved compared with traditional ASCVD score in asymptomatic patients with T2DM [26].

This study, using noninvasive radial pulse waveform and harmonic analysis, further surveyed the predictive values of the first harmonic index for the heart disease, from the symptoms and signs of CAD and the left ventricular dysfunction to the ACE (myocardial infarction, heart failure, and cardiovascular death) in a prospective cohort study. The report demonstrated that the first harmonic of radial pulse is an independent risk factor for the symptoms and signs of $\mathrm{CAD}$, the ventricular dysfunction, myocardial infarction, and heart failure. The possible mechanism could be deduced from the resonance effect of the whole arterial system, which was proposed and validated by Wang et al. [56]. The further study manifested that function of the organ can be reflected on the radial pulse spectrum [57].

The amplitude and phase of harmonic phases can be affected by arterial stiffness and the loading condition in the phantom study of the arterial system [58-60]. Consistent with the phantom study, several clinical tests also manifested that the arterial waveform revealed the status of arterial stiffness $[61,62]$ and the coronary artery perfusion [25]. Two clinical studies also found that augmentation index and augmented pressure, the calculated index of the radial pulse waveform, are independent risk markers for premature coronary artery disease [63] and for major adverse cardiac events [64] in a prospective study. Furthermore, Kingwell et al. found that the large arterial stiffness affected the arterial waveform and is associated with a reduction in myocardial contractile reserve and with myocardial ischemia symptom [65]. The above clinical study described the interactions among arterial pressure waveform, arterial stiffness, and cardiovascular events.

Wang's research shows that the first harmonic of the radial pulse wave increases with age and the augmentation index and suggests that atherosclerosis may play an important role in this age-related change of harmonics [21]. An animal study supports this concept and demonstrates that aortic stiffness in aged mice results in a significant increase in first harmonic impedance [19]. In a mice study, Segers et al. also found that the increase in cardiac afterload leads to the increase in the first harmonic impedance and the increasing need of stroke work in each heart pumping [20]. In other words, the first harmonic impedance increases along with atherosclerosis or increasing afterload, resulting in the increasing burden of heart muscle. Furthermore, an increase in the first harmonic impedance results in an increase in the harmonic amplitude of arterial pulse for the same desired stroke volume. The more stroke work increases the oxygen consumption of myocardium and may increase the risk of 
TABLE 2: Quartile of the first harmonic of the radial pulse wave as a risk for primary and secondary composite heart endpoints in 1968 type 2 diabetic patients without CAD history and angina-related symptoms. Primary composite endpoints combined the adverse heart events, including new onset of heart failure, myocardial infarction, and cardiovascular-cause mortality. Secondary composite endpoints combined the new onset of left ventricular dysfunction $(\mathrm{LVEF}<50 \%)$ and the new onset of symptoms and signs of coronary artery disease.

\begin{tabular}{|c|c|c|c|c|c|c|}
\hline \multirow{2}{*}{ Endpoint } & \multicolumn{6}{|c|}{$C 1$, first harmonic of the radial pulse wave } \\
\hline & $<0.89$ & 0.89 to 0.96 & 0.96 to 1.07 & $>1.07$ & $P$ for trend & $P$ for trend ${ }^{\#}$ \\
\hline \multicolumn{7}{|l|}{ Primary composite endpoints } \\
\hline Patients, $n(\%)$ & $37(7.5 \%)$ & $51(10.4 \%)$ & $69(14.0 \%)$ & $82(16.7 \%)$ & & \\
\hline Hazard ratio $(95 \% \mathrm{CI})$ & 1 & $\begin{array}{c}1.39 \\
(0.91-2.12)\end{array}$ & $\begin{array}{c}1.90 \\
(1.28-2.84)\end{array}$ & $\begin{array}{c}2.29 \\
(1.55-3.37)\end{array}$ & $<0.001$ & $<0.001$ \\
\hline \multicolumn{7}{|l|}{ Heart failure } \\
\hline Patients, $n(\%)$ & $15(3.0 \%)$ & $17(3.5 \%)$ & $24(4.9 \%)$ & $33(6.7 \%)$ & & \\
\hline Hazard ratio $(95 \% \mathrm{CI})$ & 1 & $\begin{array}{c}1.13 \\
(0.57-2.27)\end{array}$ & $\begin{array}{c}1.61 \\
(0.84-3.06)\end{array}$ & $\begin{array}{c}2.22 \\
(1.21-4.09)\end{array}$ & $<0.01$ & $<0.05$ \\
\hline \multicolumn{7}{|l|}{ Myocardial infarction } \\
\hline Patients, $n(\%)$ & $24(4.9 \%)$ & $37(7.5 \%)$ & $49(10.0 \%)$ & $57(11.6 \%)$ & & \\
\hline Hazard ratio $(95 \% \mathrm{CI})$ & 1 & $\begin{array}{c}1.56 \\
(0.93-2.60) \\
\end{array}$ & $\begin{array}{c}2.08 \\
(1.28-3.39) \\
\end{array}$ & $\begin{array}{c}2.44 \\
(1.51-3.93) \\
\end{array}$ & $<0.001$ & $<0.001$ \\
\hline \multicolumn{7}{|c|}{ Cardiovascular-cause mortality } \\
\hline Patients, $n(\%)$ & $2(0.4 \%)$ & $0(0 \%)$ & $2(0.4 \%)$ & $2(0.4 \%)$ & & \\
\hline Hazard ratio $(95 \% \mathrm{CI})$ & 1 & & $\begin{array}{c}1.00 \\
(0.14-7.09) \\
\end{array}$ & $\begin{array}{c}1.00 \\
(0.14-7.09) \\
\end{array}$ & NS & NS \\
\hline \multicolumn{7}{|l|}{ Secondary composite endpoints } \\
\hline Patients, $n(\%)$ & $\begin{array}{c}53 \\
(10.8 \%)\end{array}$ & $81(16.5 \%)$ & $80(16.3 \%)$ & $104(21.1 \%)$ & & \\
\hline Hazard ratio $(95 \% \mathrm{CI})$ & 1 & $\begin{array}{c}1.56 \\
(1.11-2.21)\end{array}$ & $\begin{array}{c}1.53 \\
(1.08-2.17)\end{array}$ & $\begin{array}{c}2.04 \\
(1.46-2.83)\end{array}$ & $<0.001$ & $<0.001$ \\
\hline \multicolumn{7}{|c|}{ Left ventricular dysfunction (LVEF $<50 \%)$} \\
\hline Patients, $n(\%)$ & $8(1.6 \%)$ & $11(2.2 \%)$ & $16(3.3 \%)$ & $16(3.3 \%)$ & & \\
\hline Hazard ratio $(95 \% \mathrm{CI})$ & 1 & $\begin{array}{c}1.38 \\
(0.56-3.44) \\
\end{array}$ & $\begin{array}{c}2.01 \\
(0.86-4.71)\end{array}$ & $\begin{array}{c}2.01 \\
(0.86-4.70)\end{array}$ & $<0.1$ & $<0.05$ \\
\hline \multicolumn{7}{|c|}{$\begin{array}{l}\text { Symptoms and signs of coronary artery } \\
\text { disease }\end{array}$} \\
\hline Patients, $n(\%)$ & $\begin{array}{c}51 \\
(10.4 \%)\end{array}$ & $75(15.2 \%)$ & $75(15.2 \%)$ & $100(20.3 \%)$ & & \\
\hline Hazard ratio $(95 \% \mathrm{CI})$ & 1 & $\begin{array}{c}1.49 \\
(1.05-2.13)\end{array}$ & $\begin{array}{c}1.49 \\
(1.04-2.12)\end{array}$ & $\begin{array}{c}2.03 \\
(1.45-2.84)\end{array}$ & $<0.001$ & $<0.001$ \\
\hline
\end{tabular}

The reference group for hazard ratio is the first quartile of $C 1(<0.89)$. NS = nonsignificant. ${ }^{\#} P$ for trend controlling for age, sex, smoke, systolic and diastolic blood pressure, dyslipidemia, and Hbalc.

myocardial ischemia and heart dysfunction. Therefore, this mechanism might also explain why the first harmonic of the radial artery is larger in patients with ischemic myocardium compared with controlled patients [29]. A cardiac research study supports this concept and shows that patients with heart failure had significantly higher first harmonic aortic impedance [23]. This report also showed a consistent result that the patients with a higher level of $C 1$ had a larger risk of left ventricular dysfunction, myocardial infarction, and heart failure (Table 2). However, there are many gaps among the current knowledge about the arterial pulse waveform and mechanisms of how the hemodynamic status of the ventricular-arterial system affect the pulse waveform. The further investigation is warranted to uncover the detailed relationship between the radial pulse spectrum and the risk of heart disease.

This study has some limitations. First, the radial pulse measurements were performed at baseline when enrolled patients visited the study area for the first time. Therefore, it is necessary to determine the reliabilities of the TD01C measuring instrument for research purposes. In the previous clinical study for intraobserver reliability, the population mean and standard error of the difference of $C 1$ values between successive measurements was $-0.006 \pm 0.006$ [31]. In the interobserver reliability study, the population mean, and standard error of the difference of $C 1$ values between successive measurements was $-0.002 \pm 0.008$ [31]. This showed the contribution of operator-induced bias, and variation is relatively low compared with the different levels of $C 1$ values between quartile groups. The reliability testing also demonstrated that there are no significant white coat effects for a pulse spectrum. The population mean of the difference of $C 1$ values between successive measurements within 5 minutes was close to zero. In addition, another clinical study showed that there was no significant difference in pulse spectra within 2 hours of five measurements (one measurement every 30 minutes), if only warm water was consumed before the first measurement [66]. For each same 
subject, there was no significant change in the pulse spectrum between the different measurements over the different weeks [66]. This indicated that following the standard protocol and consistent clinical procedures, the pulse wave measurements are reliable. Therefore, the white coat effects may have only a slight impact on the pulse spectrum since the subjects were well informed about the pulse spectrum measurement. Then, the results were based on a short-term prospective cohort study. The independency of $C 1$ as a cardiac risk marker only depends on about 500 events within 16 months of follow-up. More clinical studies are needed to confirm the independence of $C 1$ as a risk marker in a more general population. We have applied an extended study for 3 years of follow-up to investigate further the relationships among harmonics, traditional risk factors, and adverse cardiac events.

\section{Conclusion}

This study showed that the first harmonics amplitude of the radial pulse, $C 1$, is associated with cardiovascular events. The risk of primary and secondary composite endpoint was all increased with increasing quartile of graded $C 1$ levels. The results of the survival analysis demonstrated that the $C 1$ value is an independent predictor of ACE in asymptomatic patients with T2DM. Hence, periodic screening for radial pulse spectrum may improve the identification of type 2 diabetic patients at high risk of SCAD and confronting ACE in the future, who may need a further investigation or preventive treatment to reduce the cardiac risk.

\section{Data Availability}

The data used to support the findings of this study are available from the corresponding author upon request.

\section{Ethical Approval}

The study protocol was approved by the respective Institutional Review Board of Taipei City Hospital according to national and international regulations (IRB number: ISRCTN14306167).

\section{Consent}

All participants provided written informed consent prior to entering.

\section{Conflicts of Interest}

No potential conflicts of interest relevant to this article were reported.

\section{Authors' Contributions}

Chi-Wei Chang and Kuo-meng Liao contributed equally to this work.

\section{Acknowledgments}

This work was initiated from the collaborative project, RPWT2DM, supported by Metal Technology R \& D and Application Service Institute (RAISE10701049), Ministry of the Interior (Service1051012), and Zhongxiao Branch of Taipei City Hospital (diabetes management program). We would like to express our great appreciation for their support.

\section{References}

[1] J. Stamler, O. Vaccaro, J. D. Neaton, D. Wentworth, and Multiple Risk Factor Intervention Trial Research Group, "Diabetes, other risk factors, and 12-yr cardiovascular mortality for men screened in the multiple risk factor intervention trial," Diabetes Care, vol. 16, no. 2, pp. 434-444, 1993.

[2] X. Jouven, R. N. Lemaître, T. D. Rea, N. Sotoodehnia, J.-P. Empana, and D. S. Siscovick, "Diabetes, glucose level, and risk of sudden cardiac death," European Heart Journal, vol. 26, no. 20, pp. 2142-2147, 2005.

[3] F. J. Wackers, L. H. Young, S. E. Inzucchi et al., "Detection of silent myocardial ischemia in asymptomatic diabetic subjects: the DIAD study," Diabetes Care, vol. 27, no. 8, pp. 1954-1961, 2004.

[4] M. J. Zellweger, M. Maraun, H. H. Osterhues et al., "Progression to overt or silent CAD in asymptomatic patients with diabetes mellitus at high coronary risk: main findings of the prospective multicenter BARDOT trial with a pilot randomized treatment substudy," JACC: Cardiovascular Imaging, vol. 7, no. 10, pp. 1001-1010, 2014.

[5] K. Nademanee, V. Intarachot, M. A. Josephson, D. Rieders, F. V. Mody, and B. N. Singh, "Prognostic significance of silent myocardial ischemia in patients with unstable angina," Journal of the American College of Cardiology, vol. 10, no. 1, pp. 1-9, 1987.

[6] P. F. Cohn, "Silent myocardial ischemia: classification, prevalence, and prognosis," American Journal of Medicine, vol. 79, no. 3, pp. 2-6, 1985.

[7] L. Zednicek and J. Hruba, "Silent myocardial ischemia in diabetics," Sbornik Lekarsky, vol. 91, no. 11-12, pp. 339-345, 1989.

[8] P. Passa, C. Paillole, F. Paycha, and H. Leblanc, "Silent myocardial ischemia in diabetics. detection-prognostic and therapeutic implications," Diabete and Metabolisme, vol. 15, no. 4, pp. 206-208, 1989.

[9] W. B. Kannel and R. D. Abbott, "Incidence and prognosis of unrecognized myocardial infarction: an update on the Framingham study," New England Journal of Medicine, vol. 311, no. 18, pp. 1144-1147, 1984.

[10] E. Roelker, "Screening for coronary artery disease in patients with diabetes," Diabetes Spectrum, vol. 21, no. 3, pp. 166-171, 2008.

[11] G. A. Beller, "Noninvasive screening for coronary atherosclerosis and silent ischemia in asymptomatic type 2 diabetic patients," Journal of the American College of Cardiology, vol. 49, no. 19, pp. 1918-1923, 2007.

[12] A. J. Scholte and J. J. Bax, Screening of Asymptomatic Patients with Type 2 Diabetes Mellitus for Silent Coronary Artery Disease: Combined Use of Stress Myocardial Perfusion Imaging and Coronary Calcium Scoring, Elsevier, Amsterdam, Netherlands, 2006. 
[13] D. V. Anand, E. Lim, D. Hopkins et al., "Risk stratification in uncomplicated type 2 diabetes: prospective evaluation of the combined use of coronary artery calcium imaging and selective myocardial perfusion scintigraphy," European Heart Journal, vol. 27, no. 6, pp. 713-721, 2006.

[14] N. Rajagopalan, T. D. Miller, D. O. Hodge, R. L. Frye, and R. J. Gibbons, "Identifying high-risk asymptomatic diabetic patients who are candidates for screening stress single-photon emission computed tomography imaging," Journal of the American College of Cardiology, vol. 45, no. 1, pp. 43-49, 2005.

[15] G. Montalescot, U. Sechtem, S. Achenbach et al., "2013 ESC guidelines on the management of stable coronary artery disease: the task force on the management of stable coronary artery disease of the European society of cardiology," European Heart Journal, vol. 34, no. 38, pp. 2949-3003, 2013.

[16] D. A. McDonald, Blood Flow in Arteries, CRC Press, Boca Raton, FL, USA, 1974.

[17] W. R. Milnor, Hemodynamics, Williams \& Wilkins, Philadelphia, PA, USA, 1982.

[18] C.-H. Chen, E. Nevo, B. Fetics et al., "Estimation of central aortic pressure waveform by mathematical transformation of radial tonometry pressure. Validation of generalized transfer function," Circulation, vol. 95, no. 7, pp. 1827-1836, 1997.

[19] A. K. Reddy, Y.-H. Li, T. T. Pham et al., "Measurement of aortic input impedance in mice: effects of age on aortic stiffness," American Journal of Physiology-Heart and Circulatory Physiology, vol. 285, no. 4, pp. H1464-H1470, 2003.

[20] P. Segers, D. Georgakopoulos, M. Afanasyeva et al., "Conductance catheter-based assessment of arterial input impedance, arterial function, and ventricular-vascular interaction in mice," American Journal of Physiology-Heart and Circulatory Physiology, vol. 288, no. 3, pp. H1157-H1164, 2005.

[21] S.-H. Wang, T.-L. Hsu, M.-Y. Jan, Y.-Y. L. Wang, and W.-K. Wang, "Age-related changes in specific harmonic indices of pressure pulse waveform," in Proceedings of 13th International Conference on Biomedical Engineering, pp. 183-185, Springer, Singapore, December 2009.

[22] S.-H. Wang, W.-K. Wang, T.-L. Hsu, M.-Y. Jan, and Y.-Y. L. Wang, "Effects of captopril on specific harmonic indexes of the peripheral pressure pulse waveform," in Proceedings of 4th International Conference on Bioinformatics and Biomedical Engineering (iCBBE), pp. 1-3, IEEE, Chengdu, China, June 2010.

[23] C. J. Pepine, W. W. Nichols, and C. R. Conti, "Aortic input impedance in heart failure," Circulation, vol. 58, no. 3, pp. $460-465,1978$.

[24] J. P. Murgo, N. Westerhof, J. P. Giolma, and S. A. Altobelli, "Aortic input impedance in normal man: relationship to pressure wave forms," Circulation, vol. 62, no. 1, pp. 105-116, 1980.

[25] C. Y. Chen, W. K. Wang, T. Kao, B. C. Yu, and B. C. Chiang, "Spectral analysis of radial pulse in patients with acute, uncomplicated myocardial infarction," Japanese Heart Journal, vol. 34, no. 2, pp. 131-143, 1993.

[26] Y.-C. Chen, S. HW, G.-C. Wang, C.-W. Chang, and K.-M. Liao, "Glycated haemoglobin, ankle-brachial index, radial pulse spectrum and risk of coronary artery diseases with and without the angina symptoms in type 2 diabetic patients,", in Proceedings of International Diabetes Federation Congress, Kuala Lumpur Convention Centre (KLCC), Kuala Lumpur, Malaysia, 2018.

[27] C.-W. Chang, J.-M. Chen, and W.-K. Wang, "Radial pulse spectrum analysis as risk markers to improve the risk stratification of silent myocardial ischemia in type 2 diabetic patients," IEEE Journal of Translational Engineering in Health and Medicine, vol. 6, no. 1, 2018.

[28] K.-M. Liao, Y. cC, S.-H. Wang, M.-Y. Jan, and C.-W. Chang, "Radial pulse spectrum may be a predictor of ischemic heart disease in patients with type 2 diabetes," in Proceedings of International Diabetes Federation Congress, Abu Dhabi, United Arab Emirates, December 2017.

[29] K.-M. Liao, Y. cC, S.-H. Wang, G.-C. Wang, and C.-W. Chang, "Harmonics of the radial pulse could be risk factors for myocardial ischemia and decrease of heart function in patients with type 2 diabetes," in Proceedings of International Diabetes Federation Congress, Kuala Lumpur Convention Centre (KLCC), Kuala Lumpur, Malaysia, 2018.

[30] C.-W. Chang and W.-K. Wang, "Reliability assessment for pulse wave measurement using artificial pulse generator," Journal of Medical Engineering and Technology, vol. 39, no. 3, pp. 177-184, 2015.

[31] C.-W. Chang, J.-M. Chen, and W.-K. Wang, "Development of a standard protocol for the harmonic analysis of radial pulse wave and assessing its reliability in healthy humans," IEEE Journal of Translational engineering in health and medicine, vol. 3, pp. 1-6, 2015.

[32] J. S. Alpert, K. Thygesen, E. Antman, and J. Bassand, "Myocardial infarction redefined--a consensus document of the joint European Society of Cardiology/American College of Cardiology Committee for the redefinition of myocardial infarction," Journal of the American College of Cardiology, vol. 36, no. 3, pp. 959-969, 2000.

[33] S. A. Hunt, "ACC/AHA 2005 guideline update for the diagnosis and management of chronic heart failure in the adult: a report of the American College of cardiology/American heart association task force on practice guidelines (writing committee to update the 2001 guidelines for the evaluation and management of heart failure)," Journal of the American College of Cardiology, vol. 46, no. 6, pp. el-e82, 2005.

[34] S. A. Hunt, W. T. Abraham, M. H. Chin et al., "2009 focused update incorporated into the ACC/AHA 2005 guidelines for the diagnosis and management of heart failure in adults: a report of the American College of Cardiology Foundation/ American Heart Association task force on practice guidelines developed in collaboration with the international society for heart and lung transplantation," Journal of the American College of Cardiology, vol. 53, no. 15, pp. e1-e90, 2009.

[35] L. Zhang, H. Li, S. Zhang, L. M. Jaacks, Y. Li, and L. Ji, "Silent myocardial ischemia detected by single photon emission computed tomography (SPECT) and risk of cardiac events among asymptomatic patients with type 2 diabetes: a metaanalysis of prospective studies," Journal of Diabetes and Its Complications, vol. 28, no. 3, pp. 413-418, 2014.

[36] W. B. Kannel, "Silent myocardial ischemia and infarction: insights from the Framingham Study," Cardiology Clinics, vol. 4, no. 4, pp. 583-591, 1986.

[37] Y. Yamasaki, K. Nakajima, H. Kusuoka et al., "Prognostic value of gated myocardial perfusion imaging for asymptomatic patients with type 2 diabetes," Diabetes Care, vol. 33, no. 11, pp. 2320-2326, 2010.

[38] K. Nakajima, Y. Yamasaki, H. Kusuoka et al., "Cardiovascular events in Japanese asymptomatic patients with type 2 diabetes: a 1-year interim report of a J-ACCESS 2 investigation using myocardial perfusion imaging," European Journal of Nuclear Medicine and Molecular Imaging, vol. 36, no. 12, pp. 20492057, 2009.

[39] H. H. Feringa, S. E. Karagiannis, R. Vidakovic et al., "The prevalence and prognosis of unrecognized myocardial 
infarction and silent myocardial ischemia in patients undergoing major vascular surgery," Coronary Artery Disease, vol. 18, no. 7, pp. 571-576, 2007.

[40] Y. M. Nakao, K. Ueshima, R. Nohara et al., "Holter monitoring for the screening of cardiac disease in diabetes mellitus: the non-invasive Holter monitoring observation of new cardiac events in diabetics study," Diabetes and Vascular Disease Research, vol. 12, no. 6, pp. 396-404, 2015.

[41] M. H. Crawford, C. A. Mendoza, R. A. O'rourke, D. H. White, C. A. Boucher, and J. Gorwit, "Limitations of continuous ambulatory electrocardiogram monitoring for detecting coronary artery disease," Annals of Internal Medicine, vol. 89, no. 1, pp. 1-5, 1978.

[42] M. H. Crawford, S. J. Bernstein, P. C. Deedwania et al., "ACC/ AHA guidelines for ambulatory electrocardiography: executive summary and recommendations: a report of the American College of Cardiology/American Heart Association task force on practice guidelines (committee to revise the guidelines for ambulatory electrocardiography) developed in collaboration with the north American society for pacing and electrophysiology," Circulation, vol. 100, no. 8, pp. 886-893, 1999.

[43] M. J. Zellweger, P. Haaf, M. Maraun et al., "Predictors and prognostic impact of silent coronary artery disease in asymptomatic high-risk patients with diabetes mellitus," International Journal of Cardiology, vol. 244, pp. 37-42, 2017.

[44] S. Banerjee, A. Vinas, A. Mohammad et al., "Significance of an abnormal ankle-brachial index in patients with established coronary artery disease with and without associated diabetes mellitus," American Journal of Cardiology, vol. 113, no. 8, pp. 1280-1284, 2014.

[45] L. Potier, C. A. Khalil, K. Mohammedi, and R. Roussel, "Use and utility of ankle brachial index in patients with diabetes," European Journal of Vascular and Endovascular Surgery, vol. 41, no. 1, pp. 110-116, 2011.

[46] J. Li, Y. Luo, Y. Xu et al., "Risk factors of peripheral arterial disease and relationship between low ankle-brachial index and mortality from all-cause and cardiovascular disease in Chinese patients with type 2 diabetes," Circulation Journal, vol. 71, no. 3, pp. 377-381, 2007.

[47] Y. Choi, J. Woo, J. Kim et al., "P875 Comparison of multimodality evaluation of atherosclerosis for assessment of the severity and complexity of coronary artery disease: flowmediated dilation, ankle-brachial index and pulse wave velocity," European Heart Journal, vol. 38, no. 1, 2017.

[48] Z.-J. Zheng, A. R. Sharrett, L. E. Chambless et al., "Associations of ankle-brachial index with clinical coronary heart disease, stroke and preclinical carotid and popliteal atherosclerosis: the Atherosclerosis Risk in Communities (ARIC) Study," Atherosclerosis, vol. 131, no. 1, pp. 115-125, 1997.

[49] J. Ostergren, "Ankle brachial index is a predictor of 5-year cardiovascular events," Evidence-Based Cardiovascular Medicine, vol. 1, no. 2, p. 38, 1997.

[50] M. B. Mattock, D. J. Barnes, G. Viberti et al., "Microalbuminuria and coronary heart disease in NIDDM: an incidence study," Diabetes, vol. 47, no. 11, pp. 1786-1792, 1998.

[51] M. M. McDermott, K. Liu, M. H. Criqui et al., "Ankle-brachial index and subclinical cardiac and carotid disease: the multiethnic study of atherosclerosis," American Journal of Epidemiology, vol. 162, no. 1, pp. 33-41, 2005.

[52] J. M. Mostaza, J. R. Gonzalez-Juanatey, J. Castillo, C. Lahoz, J. M. Fernandez-Villaverde, and F. J. Maestro-Saavedra, "Prevalence of carotid stenosis and silent myocardial ischemia in asymptomatic subjects with a low ankle-brachial index," Journal of Vascular Surgery, vol. 49, no. 1, pp. 104-108, 2009.

[53] M. K. Rutter, J. M. McComb, S. Brady, and S. M. Marshall, "Silent myocardial ischemia and microalbuminuria in asymptomatic subjects with non-insulin-dependent diabetes mellitus," American Journal of Cardiology, vol. 83, no. 1, pp. 27-31, 1999.

[54] R. Demirbag, "Assessment of the relationship between silent myocardial ischemia, microalbuminuria, and left ventricular function in asymptomatic subjects with non-insulin dependent diabetes mellitus," Türk Kardiyoloji Dernĕgi Arşivi: \#x2009;Türk Kardiyoloji Dernĕginin yayın Organıdır, vol. 37, no. 2, pp. 98-100, 2009.

[55] G. Giovacchini, M. Cappagli, S. Carro et al., "Microalbuminuria predicts silent myocardial ischaemia in type 2 diabetes patients," European Journal of Nuclear Medicine and Molecular Imaging, vol. 40, no. 4, pp. 548-557, 2013.

[56] Y. Y. Wang, S. L. Chang, Y. E. Wu, T. L. Hsu, and W. K. Wang, "Resonance. The missing phenomenon in hemodynamics," Circulation Research, vol. 69, no. 1, pp. 246-249, 1991.

[57] Y. Y. L. Wang, T. L. Hsu, M. Y. Jan, and W. K. Wang, "Theory and applications of the harmonic analysis of arterial pressure pulse waves," Journal of Medical and Biological Engineering, vol. 30, no. 5, p. 335, 2010.

[58] Y. Y. L. Wang, W. K. Sze, J. G. Bau et al., "The ventriculararterial coupling system can be analyzed by the eigenwave modes of the whole arterial system," Applied Physics Letters, vol. 92, no. 15, article 153901, 2008.

[59] Y.-Y. L. Wang, W.-K. Sze, C.-C. Lin et al., "Examining the response pressure along a fluid-filled elastic tube to comprehend Frank's arterial resonance model," Journal of Biomechanics, vol. 48, no. 6, pp. 907-910, 2015.

[60] C. W. Chang, J. M. Chen, W. K. Wang, and Y. Y. Wang, "PWV measurement influenced by distance between two recording sites," American Journal of Hypertension, vol. 24, no. 3 , p. $250,2011$.

[61] W. W. Nichols, "Clinical measurement of arterial stiffness obtained from noninvasive pressure waveforms," American Journal of Hypertension, vol. 18, no. 1, pp. 3-10, 2005.

[62] S. Laurent, J. Cockcroft, L. Van Bortel et al., "Expert consensus document on arterial stiffness: methodological issues and clinical applications," European Heart Journal, vol. 27, no. 21, pp. 2588-2605, 2006.

[63] T. Weber, J. Auer, M. F. O’Rourke et al., "Arterial stiffness, wave reflections, and the risk of coronary artery disease," Circulation, vol. 109, no. 2, pp. 184-189, 2004.

[64] J. A. Chirinos, J. P. Zambrano, S. Chakko et al., "Aortic pressure augmentation predicts adverse cardiovascular events in patients with established coronary artery disease," $\mathrm{Hy}$ pertension, vol. 45, no. 5, pp. 980-985, 2005.

[65] B. A. Kingwell, T. K. Waddell, T. L. Medley, J. D. Cameron, and A. M. Dart, "Large artery stiffness predicts ischemic threshold in patients with coronary artery disease," Journal of the American College of Cardiology, vol. 40, no. 4, pp. 773-779, 2002.

[66] C.-W. Chang, S.-H. Wang, M.-Y. Jan, and W.-K. Wang, "Effect of black tea consumption on radial blood pulse spectrum and cognitive health," Complementary Therapies in Medicine, vol. 31, pp. 1-7, 2017. 


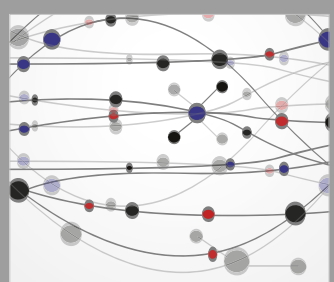

The Scientific World Journal
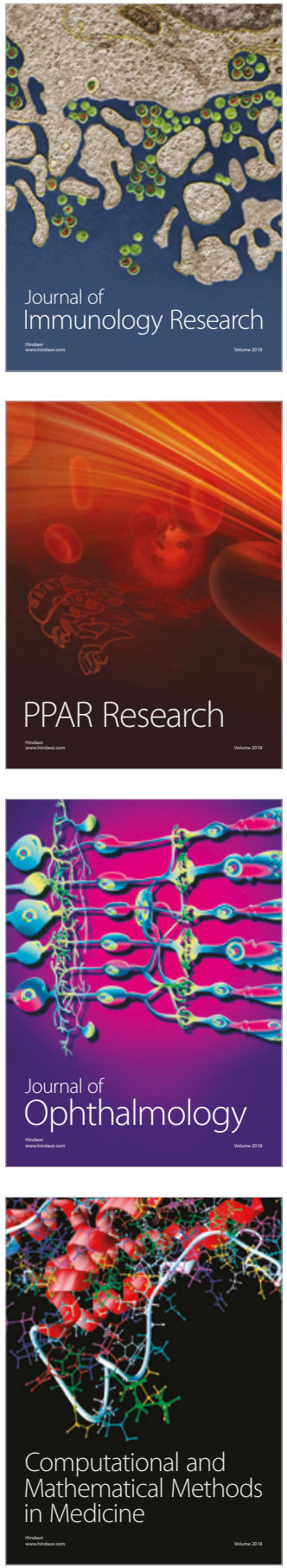

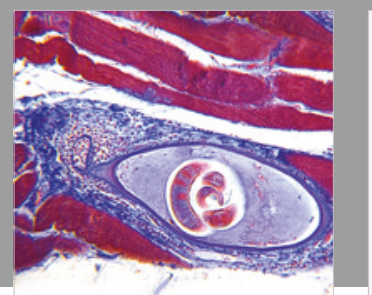

Gastroenterology Research and Practice

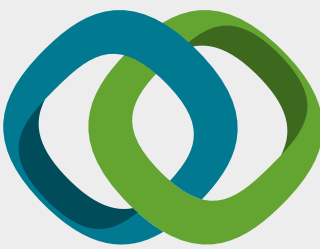

\section{Hindawi}

Submit your manuscripts at

www.hindawi.com
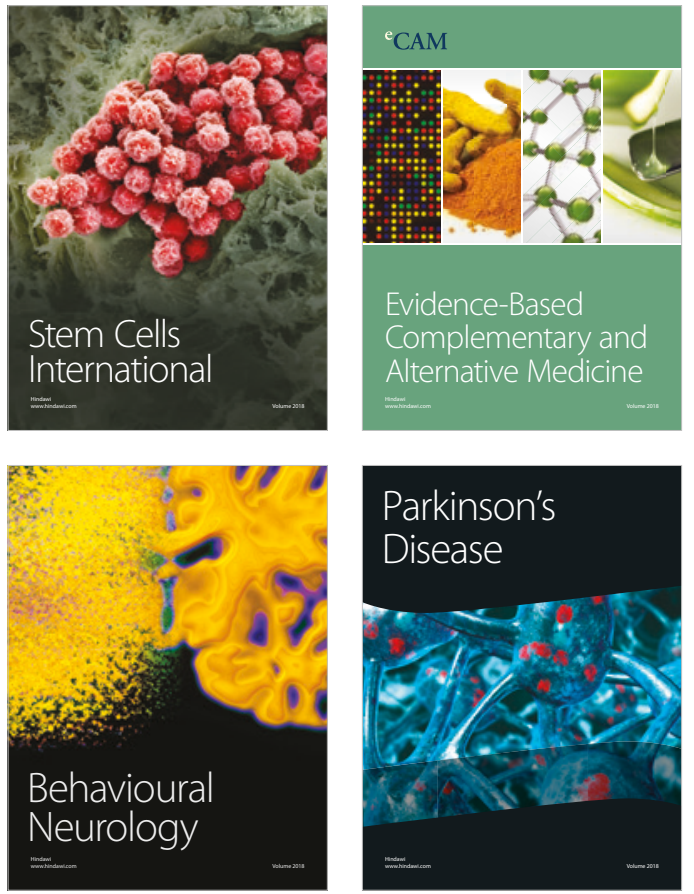

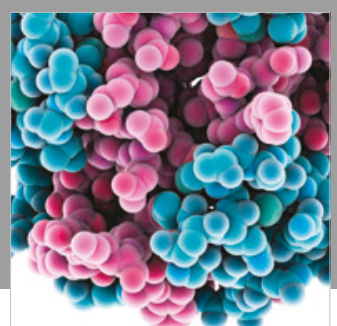

ournal of

Diabetes Research

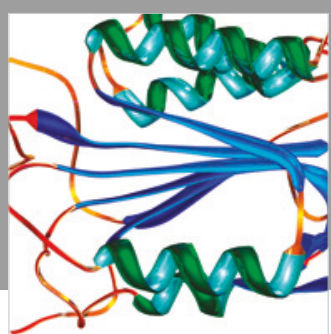

Disease Markers
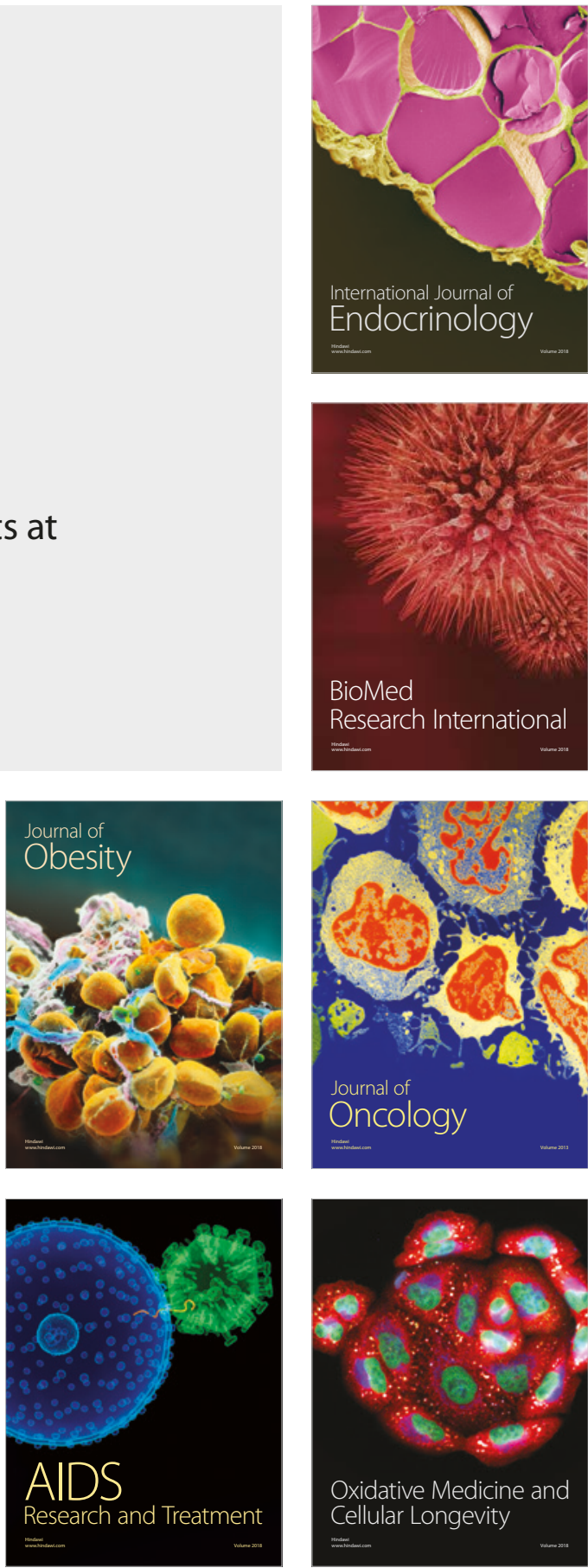